\title{
Dusty quasars eject star-forming gas from galaxies at cosmic noon
}

\author{
Hannah Stacey ( $\nabla$ hrstacey@icloud.com ) \\ Max Planck Institute for Astrophysics https://orcid.org/0000-0002-8999-9636 \\ Tiago Costa \\ Max Planck Institute for Astrophysics \\ John McKean \\ University of Groningen \\ Eilat Glikman \\ Middlebury College \\ Paul van der Werf \\ Leiden Observatory, Leiden University
}

Physical Sciences - Article

Keywords:

Posted Date: February 22nd, 2022

DOI: https://doi.org/10.21203/rs.3.rs-1355197/v1

License: (c) (1) This work is licensed under a Creative Commons Attribution 4.0 International License.

Read Full License 


\section{Dusty quasars eject star-forming gas from galaxies at cosmic noon}

3 The canonical picture of galaxy evolution invokes the injection of energy from supermassive black

4 holes to reduce star formation efficiency in massive galaxies 1 12/344. This process can explain ${ }^{566}$ their observed scaling relations, which were already established by cosmic noon (redshifts 2 to 3) ${ }^{7}$. However, the physical channels that allow energy and momentum released on sub-pc scales to affect gas on galactic scales are largely unconstrained ${ }^{8,9}$. Here we report a direct link between quasar dust-reddening and molecular outflows at $\mathrm{z} \sim 2.5$. By examining the dynamics of warm molecular gas in the inner region of galaxies, we detect outflows with velocities $700-1000 \mathrm{~km} \mathrm{~s}^{-1}$ and short timescales of 0.05 Myr that are due to ongoing quasar energy output. We observe outflows only in systems where quasar radiation pressure on dust is sufficiently large to expel their obscuring gas column densities, indicating that radiative feedback regulates gas in the nuclear regions of galaxies. This is in agreement with theoretical models that predict radiation pressure on dust in the vicinity of the black hole is a major driving mechanism of galactic-scale outflows of cold gas ${ }^{10,11,12}$. Our findings show that quasar radiation ejects star-forming gas from within nascent stellar bulges at velocities comparable to those seen on larger scales $\frac{13,14}{\text { and that }}$ molecules survive in outflows even from the most luminous quasars.

18 Type 1 quasars are characterised by broad ionic emission lines (FWHM $\left.>1000 \mathrm{~km} \mathrm{~s}^{-1}\right)$, with the 19 majority having unobscured optical continuum emission, and 20-30 percent having reddened opti$20 \mathrm{cal}_{\text {/infrared spectra }}{ }^{15}$. The distinct properties of reddened quasars indicate that they are fundamentally 21 different from their bluer counterparts $\frac{16 / 17}{}$, and it has been proposed that they represent a transitional 22 stage in quasar evolution where the obscuring dusty gas is driven out by the quasar ${ }^{17 / 18} \mid 19$. To test 23 this, we have studied a sample of fourteen Type 1 quasar hosts at $z>1$ with carbon-monoxide (CO) ${ }_{24}$ molecular line measurements at rotational level transition $J_{\text {up }} \geq 7$. Radiative transfer modelling of CO 25 line emission from AGN hosts suggests that such high excitation molecular gas must be heated by the 26 AGN radiation field because gas temperatures in star-forming regions are not high enough to produce ${ }_{27}$ their observed luminosities $20 \mid 21$. The resolved sizes of these line emitting regions are $\lesssim 1 \mathrm{kpc}^{22}$, so 28 they can be linked directly to ongoing energy and momentum release by the quasar. We use data from 
the literature and any relevant data in the Atacama Large sub-Millimetre Array (ALMA) archive with sub-arcsecond imaging to confirm that the line emission is associated with the quasar. We performed broad-band SED fitting (see Methods) to infer host galaxy star formation rate (SFR), bolometric AGN luminosity $\left(L_{\mathrm{AGN}}\right)$ and quasar extinction $\left(E_{\mathrm{B}-\mathrm{V}}\right)$, and obtained black hole masses from the literature to infer the Eddington ratios (see Methods).

We find that red quasars $\left(E_{\mathrm{B}-\mathrm{V}}>0.25^{(15}\right)$ have larger $J_{\text {up }} \geq 7 \mathrm{CO}$ line widths by a factor of $\approx 3$ when compared to blue quasars (see Fig. 1). They also inhabit a different region of parameter space with respect to $[\mathrm{CI}]$ or [CII] line widths, which primarily trace gas in the disc of the host galaxy, but have similar SFR, black hole mass and AGN luminosity to the non-red quasars. Therefore, the larger CO line widths of the red quasars cannot be explained by stronger stellar feedback, higher dynamical mass or galaxy geometry (see Fig 1 and Methods), and we conclude that these are associated with quasar-driven molecular outflows with velocities of $700-1000 \mathrm{~km} \mathrm{~s}^{-1}$. Two of the three red quasars in our sample do not appear in the NRAO VLA Sky Survey or the VLA FIRST Survey, indicating the absence of powerful radio jets and jet-mode feedback.

Two of the three red quasars in our sample (MG J0414+0534 and ULAS J1234+0907) show an excess of emission at around $3 \mu \mathrm{m}$ relative to the SED model, while none of the non-red quasars show this feature. This feature is predicted by theoretical models to be produced by thermal emission from hot dust close to the quasar sublimation radius $\frac{17 / 23}{}$. Additionally, the CO emission from the two lensed red quasars in the sample (MG J0414+0534 and J1042+1641) have been resolved and found to be $\sim 100 \mathrm{pc}$ in size (see Methods), consistent with the expected sizes of CO line emitting regions for molecular gas penetrated by strong X-ray radiation fields $24 \sqrt[25]{ }$. This confirms that the outflows we detect are within the stellar bulge ( $\lesssim 1 \mathrm{kpc}$ ), but extend beyond the region dominated by the gravity of the black holes $(5-10 \mathrm{pc})$.

In order to drive an outflow, AGN radiation pressure has to overcome the inward gravitational pull of the interstellar medium (ISM) of the host galaxy. The balance of these two forces defines an 'effective Eddington limit' 10 above which the ISM should be expelled. By relating dust extinction to column density, we show that all three red quasars lie in a 'forbidden zone' on the column density $\left(N_{\mathrm{H}}\right)$ - Eddington ratio $\left(\lambda_{\mathrm{Edd}}\right)$ plane ${ }^{11}$ (see Methods); this further supports that radiation pressure 
${ }_{57}$ is responsible for these outflows (Fig. 2). In contrast, the non-red quasars populate a region of ${ }_{58}$ the parameter space where the nucleus is unobscured, suggesting that they have already driven out their surrounding gas. Assuming a spherical model for the outflows, we infer outflow timescales of ${ }_{60} \approx 0.05 \mathrm{Myr}$ (see Methods), which are within the expected timescale of a radiatively-driven blow${ }_{61}$ out 11126 . We test an alternative scenario where the outflows are powered by an accretion disc wind, 62 but find that this mechanism is unlikely to explain the outflow velocities (see Methods). Furthermore, do not show evidence of molecular outflows (Fig. 2) demonstrating that the presence of dust is a better predictor of outflows than relativistic winds.

It has been speculated that molecules are destroyed in more energetic systems such that the outflowing gas is primarily ionised 27 . Our results show that molecules can survive in outflows, even from the brightest quasars $\left(L_{\mathrm{AGN}}>10^{48} \mathrm{ergs} \mathrm{s}^{-1}\right)$. These outflows have velocities comparable to the maximum outflow velocities of ionised gas for quasars with $L_{\mathrm{AGN}} \sim 10^{47}-10^{48} \mathrm{ergs} \mathrm{s}^{-1[27}$, and are consistent with the average outflow velocity traced by the [CII] line for one of the non-red quasars in the sample $\left(600-1000 \mathrm{~km} \mathrm{~s}^{-1}\right.$ over radii of $\left.1-5 \mathrm{kpc}^{13}\right)$. This suggests that the molecular outflow velocities are maintained as the outflow expands out of the galaxy $(\sim 10 \mathrm{kpc})$ where the cold outflow may either stall or be destroyed, transitioning to a purely ionised phase, as predicted by radiation-hydrodynamical simulations $12 \mid 28$.

State-of-the-art cosmological simulations and semi-analytic models of galaxy evolution unanimously require strong AGN feedback in order to explain the observed properties of massive galaxies across

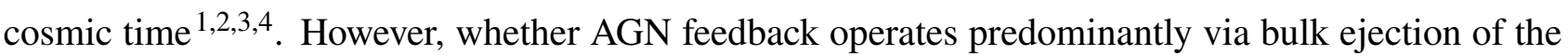
ISM or via prevention of cooling has remained an open question. We have demonstrated that quasar blow-out is effective at removing the fuel for star formation during the cosmic peak of galaxy growth, supporting an ejective feedback scenario. Due to the short outflow timescales (0.05 Myr) we are able to associate the outflows with the ongoing energy output of the quasar into the stellar bulge, finding excellent agreement with models of radiative feedback. These results indicate that radiative AGN feedback is the physical driver of scaling relations between galaxies and their central supermassive black holes 29 . 

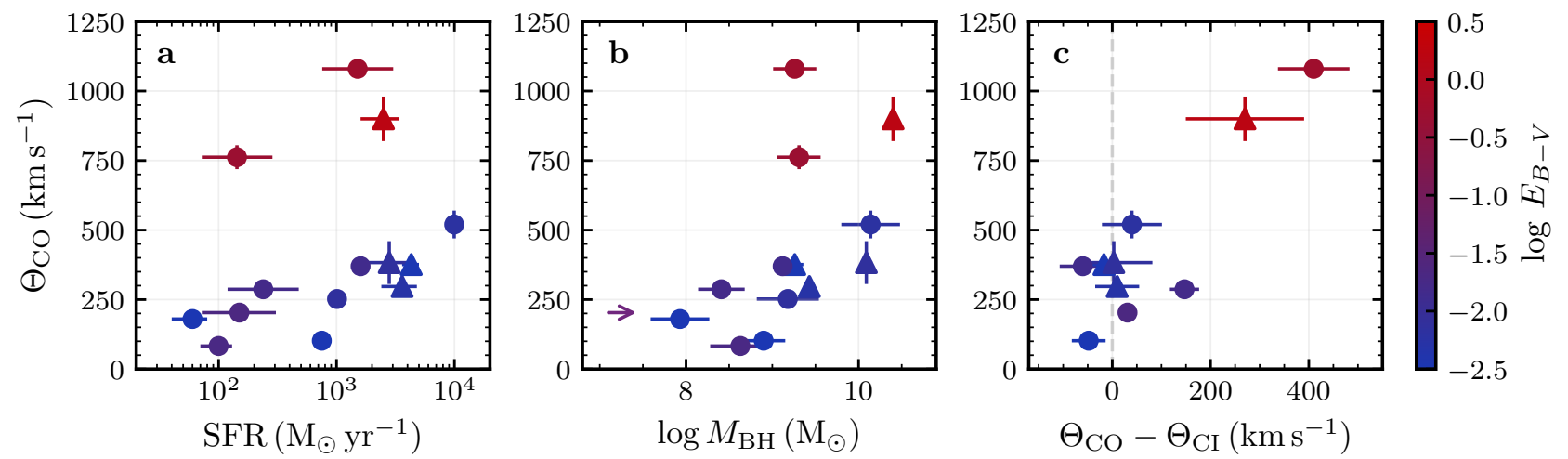

Figure 1: Comparison of CO line widths of the sample with reddening. a: $J_{\text {up }}>7 \mathrm{CO}$ FWHM against SFR (lensing-corrected). b: CO FWHM against $M_{\mathrm{BH}}$ (lensing-corrected). c: difference in $\mathrm{CO}$ and $[\mathrm{CI}]$ or $[\mathrm{CII}]$ FWHM (as a proxy for galaxy dynamical mass) against CO FWHM. The circles identify the sources that are lensed. The colour scale indicates quasar extinction $\left(E_{\mathrm{B}-\mathrm{V}}\right)$. The red quasars inhabit a different parameter space than the blue quasars, which follow expected trends with dynamical mass (see Methods). 


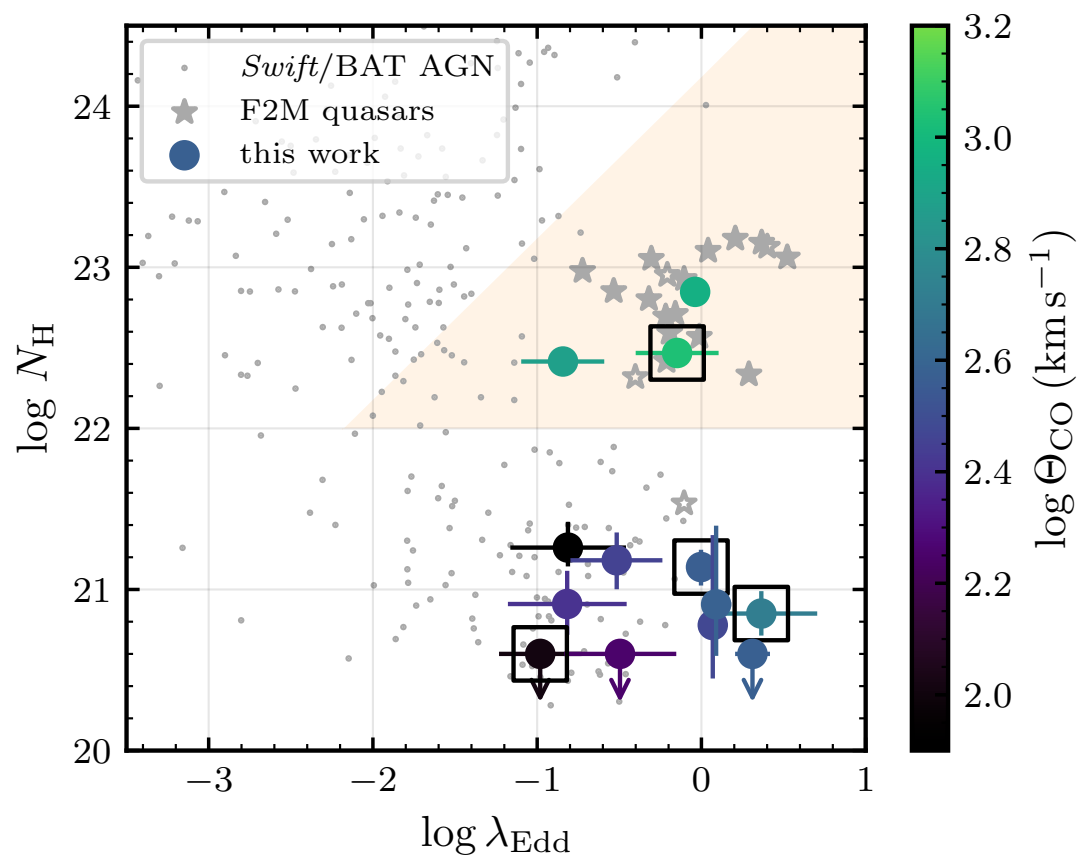

Figure 2: Eddington ratio against gas column density. The shaded region identifies the 'forbidden zone' where radiation pressure on dust is sufficient to expel the obscuring column density ${ }^{11}$ (see Methods). We convert dust-reddening $\left(E_{\mathrm{B}-\mathrm{V}}\right)$ to gas column density $\left(N_{\mathrm{H}}\right)^{\frac{30}{2}}$, assuming that the molecular gas is a good approximation for the total gas column density. We cannot reliably constrain $E_{\mathrm{B}-\mathrm{V}}$ at values below 0.01 , so adopt this as an upper limit for the equivalent column density. Boxes identify the objects in our sample where ultra-fast outflows or broad absorption lines have been detected 31 . The stars show quasars from F2M surveys $\left(0.1<E_{\mathrm{B}-\mathrm{V}}<2.5\right)$ where $N_{\mathrm{H}}$ is inferred from $E_{\mathrm{B}-\mathrm{V}}\left(\right.$ filled stars) or X-ray spectra (open stars) ${ }^{32}$. The dots are X-ray detected AGN in the Swift/BAT sample $\mathrm{e}^{33}$, where $N_{\mathrm{H}}$ is inferred from X-ray spectra. The red quasars are in the forbidden zone where radiative pressure can drive out the obscuring gas, indicating that radiation pressure is responsible for the outflows. 


\section{${ }_{85}$ Methods}

\section{ALMA observations}

${ }_{87}$ In addition to $\mathrm{CO},[\mathrm{CI}]$ and $[\mathrm{CII}]$ measurements obtained from the literature, we present new ALMA 88 data for several objects. These observations are summarised in Table 1 . Where we observe the 89 $\mathrm{CO}(7-6)$ lines we also observe the $[\mathrm{CI}](2-1)$ line simultaneously. Each data set was calibrated and 90 reduced using the ALMA pipeline in $\mathrm{CASA}^{34}$. The calibrated data were inspected to confirm the 91 quality of the pipeline calibration. We self-calibrated the data for SDSS J0248+1913 and

92 SDSS J1330+1810 using the continuum-only spectral windows with a single solution interval for ${ }_{93}$ each antenna in both amplitude and phase. For MG J0414+0534, we performed phase calibration with a solution interval of 240 seconds.

We created a clean image cube of the spectral window containing the line emission with natural ${ }_{96}$ weighting of the visibilities (Fig. 3) and extracted a spectrum in an aperture around the lens. The line 97 profiles (presented here and where taken from the literature) do not show asymmetries that would indicate that their shapes are strongly affected by differential magnification.

99

To measure the line FWHM, we fit the line profiles with a single Gaussian. For MG J0414+0534, the CO (7-6) line is so broad that it is blended with the [CI] (2-1) line (Fig. 4r). We fit Gaussians to both lines simultaneously with the systemic redshift fixed to the value found for the $\mathrm{CO}(3-2)^{35}$. For SDSS J1330+1810, part of the [CI] line falls outside the spectral window. For these two cases, the integrated line luminosity is estimated from the fitted Gaussian profile rather than directly from the image. The line profiles and Gaussian fits for these ALMA observations are shown in Fig. 4 .

\section{AGN luminosity, extinction and black hole masses}

We use AGNFITTER ${ }^{36}$ to fit the broad-band SED of each quasar and host galaxy (Fig. 5). This software includes accretion disc, torus, host galaxy and starburst templates $\frac{1736}{2}$. The accretion disc component is reddened by an extinction model ${ }^{37}$ parameterised by rest-frame $E_{B-V}$. We compile photometry from all-sky surveys, where available, or from the literature.

We assume a minimum uncertainty of 10 percent on photometric measurements to allow for source variability and microlensing effects. Although the photometry will be blended with light from the 
lensing galaxy, the quasar point-source emission strongly dominates the emission. This is evident from the available ultra-violet-infrared imaging with the Hubble Space Telescope (HST), e.g. in the CfA-Arizona Space Telescope Lens Survey $\frac{38}{3}$. The host galaxy emission can be fit in only a few cases, for most only accretion disc and torus components are constrained.

Following previous work ${ }^{17}$, we calculate the bolometric AGN luminosity $\left(L_{\mathrm{AGN}}\right)$ by integrating the quasar accretion disc model ('big blue bump') in the range $0.05-1 \mu \mathrm{m}$ with an additive correction of 0.3 dex to account for X-ray emission not included in the model fit, i.e.

$$
\log L_{\mathrm{AGN}}=\log L_{0.05-1 \mu \mathrm{m}}+0.3-\log \mu_{\mathrm{qso}},
$$

where $\mu_{\mathrm{qso}}$ is the quasar magnification. We assume black hole mass $\left(M_{\mathrm{BH}}\right)$ estimates from the literature that were derived using the virial method. For consistency, we adopt the same quasar lensing magnification used for the black hole mass to infer the intrinsic bolometric AGN luminosity.

\section{Star formation rates}

Where possible, we obtain far-infrared luminosities from the literature. While AGNFITTER includes templates to fit the host galaxy dust emission, these models are empirically derived from low-redshift starbursts and may not be appropriate for high-redshift quasar hosts. Indeed, we found that the far-infrared-mm spectra are often poorly fit by these templates. Instead, we adopt far-infrared luminosities taken from the literature, most of which were calculated with free-form models. Where there is insufficient photometry to fit a dust model, we assume an optically-thin modified black-body with the median effective dust temperature and emissivity found for a large sample of quasar hosts 39 . While the effective dust temperature may be quite different depending on the choice of model, the total infrared luminosity will be similar as it is constrained by the photometry.

We convert far-infrared luminosity to total infrared luminosity $(8-1000 \mu \mathrm{m})$ using the colour-correction factor $^{40}$ of 1.91 and to a dust-obscured SFR using a standard calibration ${ }^{41}$ assuming a Salpeter initial mass function.

The intrinsic SFR for some of the lensed sources have previously been inferred from lens modelling of ALMA observations of sub-mm dust emission ${ }^{22}$. For APM $08279+5255$, we adopt the CO 
magnification factor from lens modelling in literature to infer a SFR from the uncorrected measurement ${ }^{42}$. For other lensed sources, we assume a sub-mm magnification factor of $10_{-5}^{+10}$ in line with typical values for four-image lensed quasar systems ${ }^{22}$ to infer the SFR. All values and references are given in Table 2 .

Note that the sub-mm magnification is not the same as the quasar magnification, as these will be lensed differently depending on the size and location of the emitting region. We did not check for consistency between the lens models used to obtain black hole masses from the literature and those used to obtain the SFR, but we do not compare these properties.

\section{Comparison of CO line dynamics}

Details of the line properties, SFR, black hole masses, $E_{\mathrm{B}-\mathrm{V}}$, AGN luminosities and literature references for the sample of quasars is presented in Table 2. The sample probes 2, 3 and 4 orders of magnitude in SFR, black hole mass and AGN luminosity, respectively.

The sample probes a range of CO rotational transitions, $J_{\text {up }}=7-11$. The kinematics of these high-excitation CO lines have not yet been systematically studied at high redshift so little is known about their typical dynamics. However, we are able to compare the $\mathrm{CO}(7-6)$ and $\mathrm{CO}(11-10)$ line widths for MG J0414+0534 and find comparably large values (Table 2 These lines are both significantly broader than the $580 \mathrm{~km} \mathrm{~s}^{-1}$ found for the $\mathrm{CO}(3-2)$ line $\mathrm{e}^{\sqrt{35}}$ in the same system, suggesting the $\mathrm{CO}(7-6)$ has similar dynamics to higher excitation lines.

For the non-red quasars, we find positive correlations between CO FWHM and SFR $(p=0.004)$ and between CO FWHM and black hole mass $(p=0.00001)$. While Pearson correlation tests may not be accurate when considering such small sample sizes, these correlations can be explained by well-understood physical phenomena. The correlation with SFR (Fig. 1, top) could reflect increased turbulence due to radiative stellar winds or supernovae: gas dispersion of up to $100 \mathrm{~km} \mathrm{~s}^{-1}$ can be induced by stellar feedback in regions of Eddington-limited star formation ${ }^{43}$. Alternatively, it may reflect the relationship between SFR and dynamical mass. The correlation between FWHM and black hole mass (Fig. 1, middle) may reflect a larger dynamical mass as expected from canonical scaling relations between black hole mass and stellar mass ${ }^{29}$. These are expected correlations found

\footnotetext{
${ }^{\dagger}$ The FWHM of the CO(11-10) line is shown for MG J0414+0534 in all plots
} 
for $[\mathrm{CII}]$ in quasar host galaxies $\frac{45}{4}$, although these have not been previously investigated for these high-excitation CO lines. The red quasars do not follow the correlations observed by the non-red quasars, which follow expected trends with dynamical mass, and there appears to be no significant correlation between SFR or black hole mass and reddening ( $p=0.45$ and $p=0.24$, respectively, for the whole sample).

We also compare the high-excitation $\mathrm{CO}$ with $[\mathrm{CI}]$ or $[\mathrm{CII}]$ line profiles to further test whether the $\mathrm{CO}$ lines are associated with the host galaxy disc dynamics or inclination. $[\mathrm{CI}]$ and $[\mathrm{CII}]$ trace gas at lower temperatures and densities, but also CO-dark molecular gas $\frac{46}{4}$, so they probe the galaxy at larger disc radii (i.e. maximum circular velocity) ${ }^{45 / 47 / 48}$. Fig. 1 (bottom) shows the CO FWHM against its difference from the $[\mathrm{CI}]$ or $[\mathrm{CII}]$ line FWHM for the sources with such measurements. The $\mathrm{CO}$ and $[\mathrm{CI}]$ or $[\mathrm{CII}]$ line FWHMs are similar for the blue quasars, so these lines could both relate to gas in the host galaxy. However, the red quasars have CO FWHMs $300-400 \mathrm{~km} \mathrm{~s}^{-1}$ larger than their $[\mathrm{CI}]$ or $[\mathrm{CII}]$ line widths, indicating that the $\mathrm{CO}$ is not primarily from rotating gas in the disc. Therefore, we conclude that outflows are the most likely explanation for the CO large linewidths. Using an orthogonal distance regression, we fit a linear relationship between CO FWHM and SFR for the non-red quasars. Assuming the line profiles have contributions from both outflows and host galaxy which can be described by Gaussians of equal height, we estimate a line-of-sight outflow velocity of $1030 \pm 80,750 \pm 50$ and $820 \pm 140 \mathrm{~km} \mathrm{~s}^{-1}$ for MG J0414+0534, J1042+1641 and ULAS J1234+0907, respectively. These velocities may be intrinsically larger depending on the geometry of the outflows.

As we show in Figs. 2 and 6, there is no indication that the red quasars in our sample have larger AGN luminosities or larger Eddington ratios than the non-red quasars (although this may not be true for the populations in a statistical sense ${ }^{49}$ ).

\section{Radiatively-driven outflows}

The abundance of dust is conducive to strong quasar feedback. By enhancing the absorption cross section to ultra-violet and optical AGN radiation, the dusty ISM can, in principle, be driven out by radiation pressure ${ }^{10 / 11}$. The balance of these two forces defines an 'effective Eddington limit' 10 
above which the ISM can be expelled, resulting in correlations between galaxy bulge properties and black hole mass that are in agreement with observations 50 .

Recent radiation-hydrodynamic simulations $\frac{12[28 \mid 51}{}$ predict that radiation pressure operates and launches outflows from $<1 \mathrm{kpc}$ scales, which shock-heat and generate hot, over-pressurised bubbles on larger scales ${ }^{52}$ thereby reducing the supply of gas for star formation in the inner region of the galaxy. This blow-out phase is likely short-lived because outflows propagate on a characteristic timescale of

$$
t_{\text {out }} \sim 1\left(R_{\text {out }} / \mathrm{kpc}\right)\left(v_{\text {out }} / 1000 \mathrm{~km} \mathrm{~s}^{-1}\right)^{-1} \mathrm{Myr} .
$$

For this reason, we expect quasars to be below the effective Eddington limit unless their host galaxies are experiencing a powerful outflow. This limit creates a 'forbidden zone' on the $\lambda_{\mathrm{Edd}}-N_{\mathrm{H}}$ plane in the regime where the dust is optically thick to ultra-violet radiation which can approximated by

$$
N_{\mathrm{H}} \sim \frac{\lambda_{\mathrm{Edd}} / \sigma_{\mathrm{T}}}{1-\frac{\kappa_{\mathrm{IR}}}{\kappa_{\mathrm{T}}} \lambda_{\mathrm{Edd}}},
$$

where $\sigma_{\mathrm{T}}$ is the Thompson cross-section, $\kappa_{\mathrm{T}}$ is the electron scattering opacity and $\kappa_{\mathrm{IR}}$ is the dust opacity 11 . Previous studies ${ }^{1153}$ have defined lower limits for the forbidden zone of $10^{21.5}-10^{22} \mathrm{~cm}^{-2}$ where column densities could be associated with diffuse cold gas in the galaxy: we have adopted the more conservative assumption of $10^{22} \mathrm{~cm}^{-2}$, although the choice does not affect our interpretation.

Two red quasars in our sample have measured sizes of their $\mathrm{CO}$ emission from lens modelling in the literature ${ }^{54}$ and this work (Fig. 7). From Eq. 2, we estimate an outflow timescale of $\approx 0.05 \mathrm{Myr}$ for MG J0414+0534 and J1042+1641

\section{Test of an energy-driven scenario}

Fig. 2 supports the scenario that radiation pressure drives the observed outflows. However, outflows may also be ‘energy-driven' by high-velocity winds produced near the accretion disc 6 52 [55|56 Ultra-fast outflows have been detected in X-ray spectra of high-redshift quasars $\frac{57}{1 n c l u d i n g}$ the red quasar MG J0414+0534, which contains an ultra-fast outflow with velocity $0.3 c^{\frac{58}{1}}$ that could potentially power the observed molecular outflow. The kinematics of the wind is a key factor to discriminate between the two scenarios: the momentum flux for energy-driven outflows is expected 
to be $\dot{p} \sim v_{\text {out }} / v_{\mathrm{UFO}} \gtrsim 100 L_{\mathrm{AGN}} / c$, compared to $\dot{p} \sim L_{\mathrm{AGN}} / c$ for momentum or radiation pressure-driven winds $\frac{12}{12}$. For the inferred $L_{\mathrm{AGN}}=10^{47.2} \mathrm{ergs} \mathrm{s}^{-1}$, we predict an energy-driven mass outflow rate $\left(\dot{M}=\dot{p} / v_{\text {out }}\right)$ of $\approx 10^{6} \mathrm{M}_{\odot} \mathrm{yr}^{-1}$ for the gas probed by the $\mathrm{CO}(11-10)$. Adopting a simple spherical model for the molecular outflow with radius of $\approx 50 \mathrm{pc}^{54}$ and velocity $1000 \mathrm{~km} \mathrm{~s}^{-1}$, the implied outflow timescale is $\approx 0.05 \mathrm{Myr}$ and thus its total mass is $\approx 10^{10} \mathrm{M}_{\odot}$. Adopting the $3 \sigma$ upper limit for non-detection of $\mathrm{CO}(1-0)^{\sqrt[59]{ }}$, a typical CO-to- $\mathrm{H}_{2}$ conversion factor of $0.8 \mathrm{~K} \mathrm{~km} \mathrm{~s}^{-1} \mathrm{pc}^{2}$ and typical ${ }^{39}$ magnification factor of 10 , the molecular gas mass in the host galaxy is $<10^{9.5} \mathrm{M}_{\odot}$. Therefore, for an energy-driven wind model, the gas in the outflow from MG J0414+0534 would be more than twice as massive as the molecular gas in the disc: an unlikely scenario given the high SFR (Table 2) and the massive molecular gas reservoirs of quasar hosts that are similar to normal starbursts 59|60|61. This disfavours an energy-driven scenario for this object, implying that an accretion disc wind must couple very inefficiently with the ISM in order to produce the outflow we observe. 


\section{Acknowledgements}

${ }_{228}$ This research used SciPy, NumPy and Matplotlib packages for Python ${ }^{62 \mid 63 / 64}$, and also the

229 NASA/IPAC Extragalactic Database (NED), SIMBAD ${ }^{65}$ and VizieR ${ }^{66}$ catalogue access tools. We 230 made use of ALMA data with project codes 2018.1.01008.S, 2019.1.00948.S and 2019.1.00964.S.

${ }_{231}$ ALMA is a partnership of ESO (representing its member states), NSF (USA) and NINS (Japan), ${ }_{232}$ together with NRC (Canada), MOST and ASIAA (Taiwan), and KASI (Republic of Korea), in ${ }_{233}$ cooperation with the Republic of Chile. The Joint ALMA Observatory is operated by ESO, ${ }_{234}$ AUI/NRAO and NAOJ.

\section{${ }_{235}$ Data Availability}

${ }_{236}$ All observations reported in this work are publicly available in the ALMA archive

${ }_{237}$ (https://almascience.eso.org/aq). The data sets generated during the current study are ${ }_{238}$ available from the corresponding author on reasonable request. 

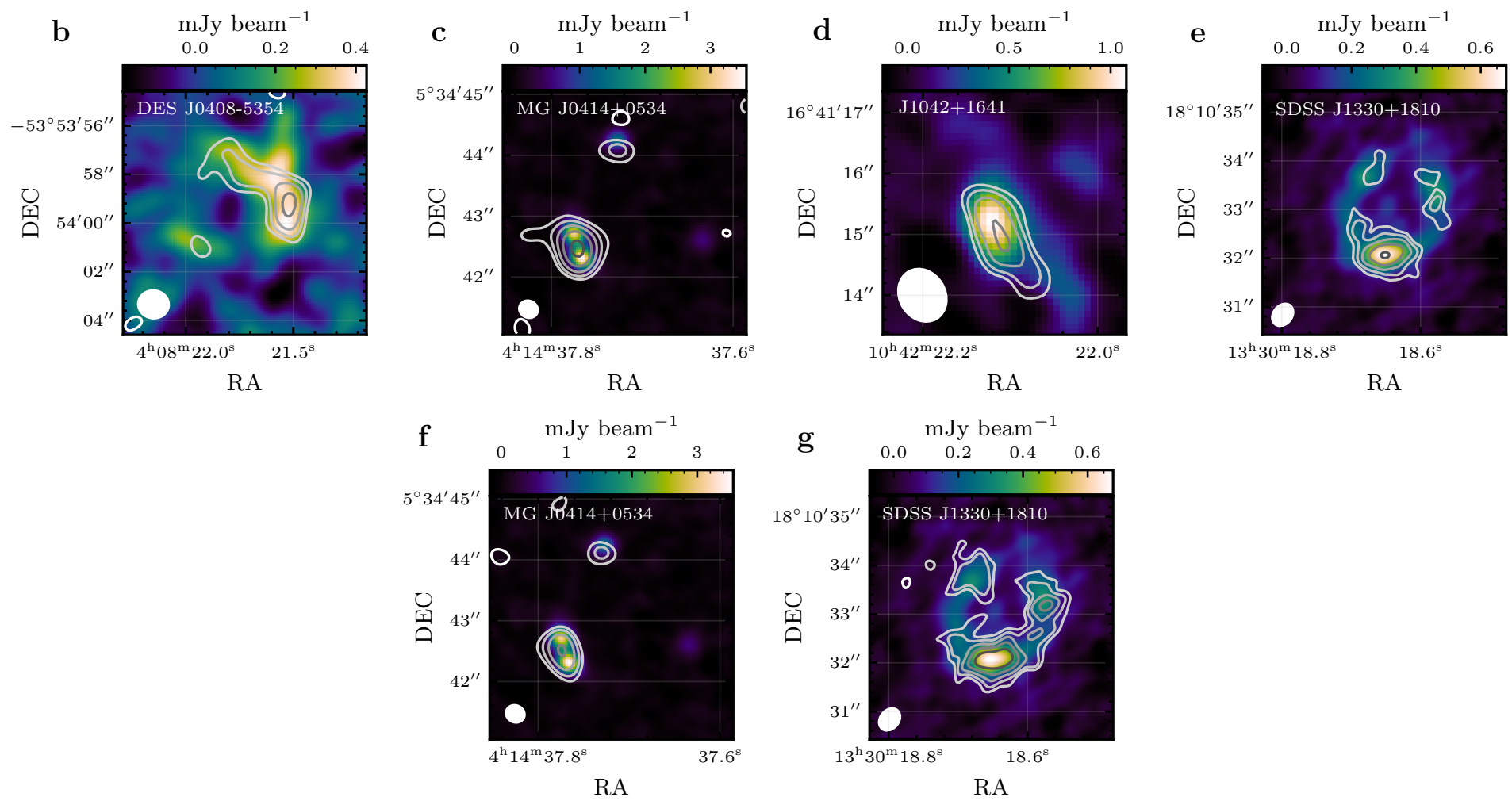

Figure 3: ALMA imaging for the new data presented in this work. a-d: the sub-mm continuum emission with contours of the velocityintegrated CO line emission in signal-to-noise intervals of $-3,3,3 \sqrt{2}, 6,6 \sqrt{2}$.. etc. The synthesised beam is shown by the ellipse in the lower-left corner. e,f: the same for the $[\mathrm{CI}]$ line emission. 

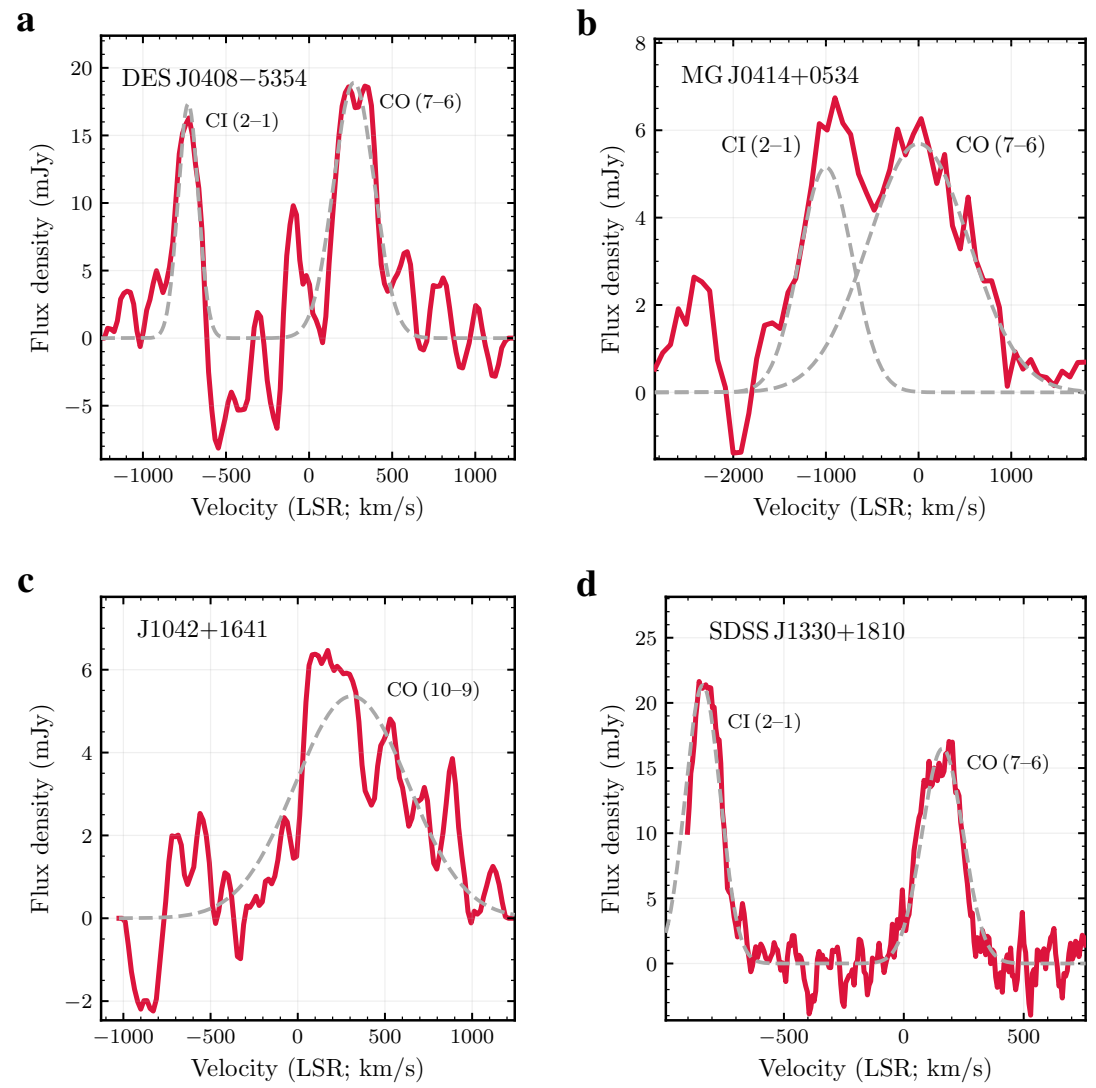

Figure 4: Line profiles for the new data presented in this work. The spatially-integrated line profiles (red line) and Gaussian fits to the $\mathrm{CO}$ and $[\mathrm{CI}]$ line emission (grey dashed line). For all except MG J0414+0534 (c), the systemic redshift is based on optical spectroscopy so a velocity offset in the CO could be because the ionic lines trace out-flowing gas. 

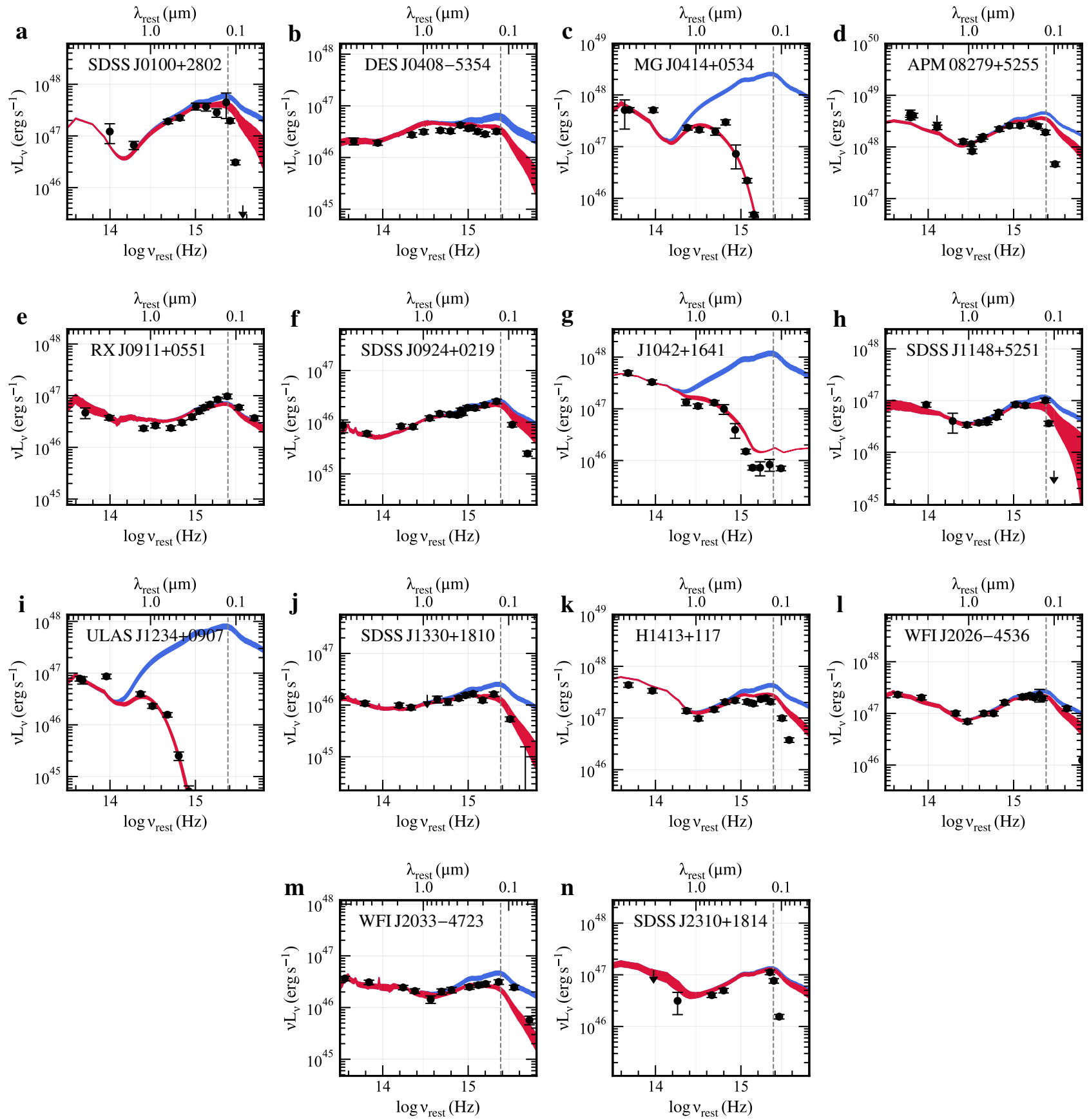

Figure 5: SED fits for the quasar sample. We use AGNFitter ${ }^{36}$ to fit broad-band SEDs from the ultra-violet to mid-infrared. The red curve is a solid fill between 16th to 86th percentiles of the models from the MCMC analysis. The blue curve shows the quasar models without dust attenuation. The grey dashed line is the rest-frame frequency of Lyman- $\alpha$; photometric measurements at or above this frequency are ignored because they are affected by absorption by the intergalactic medium. 


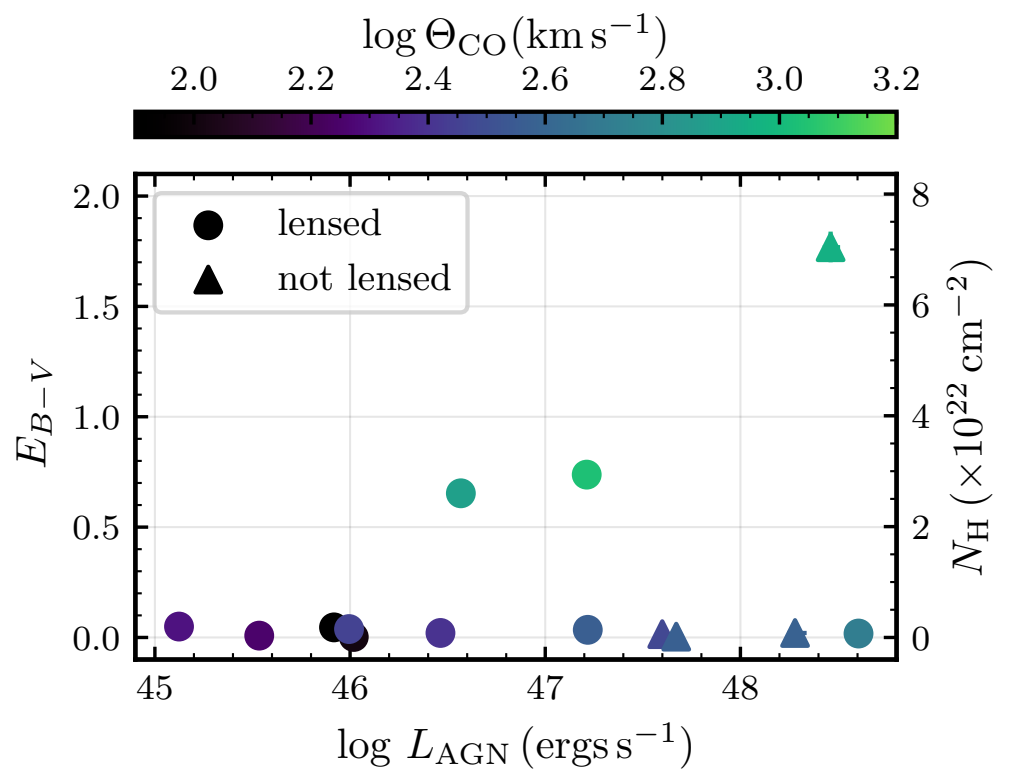

Figure 6: Reddening against bolometric AGN luminosity (lensing-corrected). The opposing y-axis shows the corresponding column density 30 . Circles identify sources that are lensed. Outflows are observed even for AGN luminosities $>10^{48} \mathrm{ergs} \mathrm{s}^{-1}$.
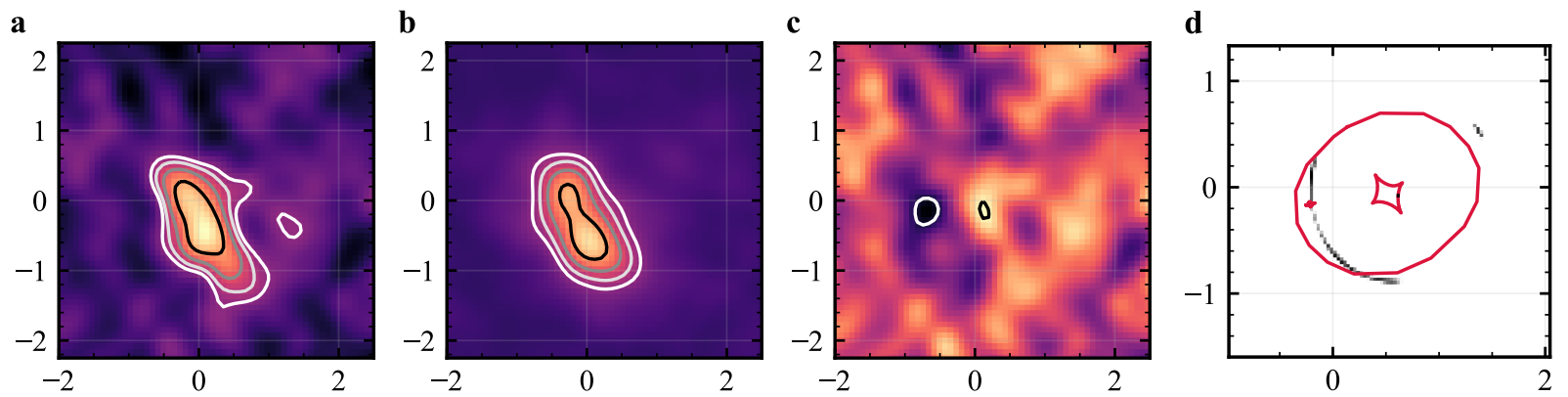

Figure 7: Lens modelling of J1042+1641. We use visILENs $\frac{67 \mid 68}{6}$ to model the integrated CO (10-9) emission. We adopt the fiducial lens model from the literature ${ }^{15}$ consisting of a singular isothermal ellipsoid for the main lens, an isothermal sphere for the satellite galaxy and external shear. The source is modelled as a circular Gaussian with size, position and flux density as free parameters. We find a best-fit FWHM of $70 \pm 10$ pc. a-c: The dirty image of the data, dirty image of the model and residual image. The contours are in signal-to-noise ratio intervals of $-3,3,3 \sqrt{2}, 6,6 \sqrt{2}$. d: The source and lens model (grey; log-scale) and lensing caustics (red). 


\begin{tabular}{llccccc}
\hline Project & Name & Line $(\mathrm{s})$ & $\begin{array}{c}\Theta_{\text {beam }} \\
(\operatorname{arcsec})\end{array}$ & $\begin{array}{c}v_{\text {cont }} \\
(\mathrm{GHz})\end{array}$ & $\begin{array}{c}S_{v} \\
(\mathrm{mJy})\end{array}$ & $\begin{array}{c}I_{\text {line }} \\
\left(\mathrm{Jy} \mathrm{km} \mathrm{s}^{-1}\right)\end{array}$ \\
\hline 2018.1.01008.S & MG J0414+0534 & $\mathrm{CO}(7-6) ;[\mathrm{CI}](2-1)$ & $0.3 \times 0.3^{\dagger}$ & 236 & $10.1 \pm 0.4$ & $7.8^{*} ; 3.7^{*}$ \\
2019.1.00948.S & SDSS J1330+1810 & $\mathrm{CO}(7-6) ;[\mathrm{CI}](2-1)$ & $0.5 \times 0.4$ & 342 & $6.3 \pm 0.2$ & $3.5 \pm 0.2 ; 3.9^{*}$ \\
2019.1.00964.S & DES J0408-5354 & $\mathrm{CO}(7-6) ;[\mathrm{CI}](2-1)$ & $1.2 \times 1.1^{\dagger}$ & 247 & $3.0 \pm 0.4$ & $4.9 \pm 0.7 ; 3.3 \pm 0.5$ \\
& J1042+1641 & $\mathrm{CO}(10-9)$ & $0.9 \times 0.7$ & 256 & $2.0 \pm 0.3$ & $4.0 \pm 0.7$ \\
\hline
\end{tabular}

Table 1: New ALMA observations presented in this work. We give the project code, the synthesised beam FWHM $\left(\Theta_{\mathrm{FWHM}}\right)$ used to extract the line profile and continuum flux density, the continuum frequency $\left(v_{\text {cont }}\right)$, the continuum flux density $\left(S_{v}\right)$, the integrated line intensity $\left(I_{\mathrm{CO}}\right)$ and line systemic redshift $\left(z_{\mathrm{CO}}\right)$ based on a Gaussian fit to the line profile. $\dagger$ denotes cases where we have applied a $u v$ taper to the data weights to improve surface brightness sensitivity. ${ }^{*}$ For these cases, the line intensity is approximated from the Gaussian fit to the line profile (see Methods). 


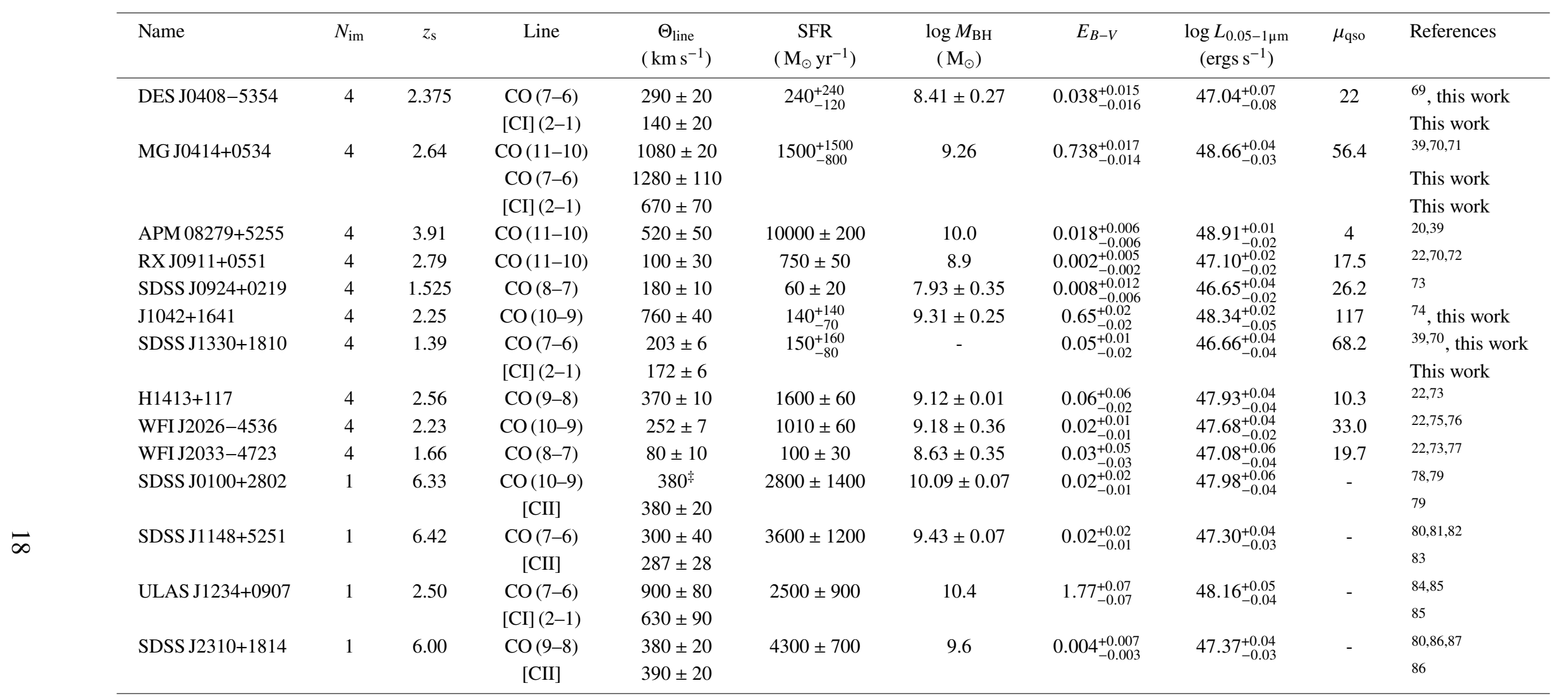

Table 2: Summary of the objects in the sample. We give the number of lensed quasar images $\left(N_{\mathrm{im}}=1\right.$ if not strongly lensed $)$, source redshift $\left(z_{s}\right)$, CO line transition (or other line), line FWHM from a single Gaussian fit to the line profile, SFR (lensing-corrected), black hole mass $\left(M_{\mathrm{BH}}\right)$, extinction $\left(E_{B-V}\right)$, integrated luminosity of the BBB template ( $\log L_{0.05-1 \mu \mathrm{m}}$, not lensing-corrected) and assumed value of the quasar magnification from the literature $\left(\mu_{\mathrm{qso}}\right)$. Where no uncertainty was given for the black hole mass, we assume an uncertainty of 0.25 dex which is the typical scatter found for black hole scaling relations. For SDSS J1330+1810, line widths have not been reported, so we infer a lower limit the black hole mass from the AGN bolometric luminosity assuming Eddington-limited accretion. References given are for the line FWHM, black hole mass and quasar magnification, and photometry used for the optical-infrared SED fitting (if not from all-sky catalogues). 


\section{References}

[1] Di Matteo, T., Springel, V. \& Hernquist, L. Energy input from quasars regulates the growth and activity of black holes and their host galaxies. Nature 433, 604-607 (2005). astro-ph/0502199

[2] Somerville, R. S. \& Davé, R. Physical Models of Galaxy Formation in a Cosmological Framework. ARA\&A 53, 51-113 (2015). 1412.2712

[3] Schaye, J. et al. The EAGLE project: simulating the evolution and assembly of galaxies and their environments. MNRAS 446, 521-554 (2015). 1407.7040.

[4] Weinberger, R. et al. Simulating galaxy formation with black hole driven thermal and kinetic feedback. MNRAS 465, 3291-3308 (2017). 1607.03486.

[5] King, A. The AGN-Starburst Connection, Galactic Superwinds, and $\mathrm{M}_{B H^{-}}-\sigma$. ApJL 635, L121-L123 (2005). astro-ph/0511034.

[6] Costa, T., Sijacki, D. \& Haehnelt, M. G. Feedback from active galactic nuclei: energy- versus momentum-driving. MNRAS 444, 2355-2376 (2014). 1406.2691.

[7] Förster Schreiber, N. M. \& Wuyts, S. Star-Forming Galaxies at Cosmic Noon. ARA\&A 58, 661-725 (2020). 2010. 10171.

[8] Harrison, C. M. Impact of supermassive black hole growth on star formation. Nature Astronomy 1, 0165 (2017). 1703.06889.

[9] Harrison, C. M. et al. AGN outflows and feedback twenty years on. Nature Astronomy 2, 198-205 (2018). 1802.10306.

[10] Fabian, A. C., Vasudevan, R. V. \& Gandhi, P. The effect of radiation pressure on dusty absorbing gas around active galactic nuclei. MNRAS 385, L43-L47 (2008). 0712.0277.

[11] Ishibashi, W., Fabian, A. C., Ricci, C. \& Celotti, A. Revisiting the 'forbidden’ region: AGN radiative feedback with radiation trapping. MNRAS 479, 3335-3342 (2018). 1806.08387. 
[12] Costa, T., Rosdahl, J., Sijacki, D. \& Haehnelt, M. G. Quenching star formation with quasar outflows launched by trapped IR radiation. MNRAS 479, 2079-2111 (2018). 1709.08638.

[13] Cicone, C. et al. Very extended cold gas, star formation and outflows in the halo of a bright quasar at $\mathrm{z}>6$. A\&A 574, A14 (2015). 1409.4418 .

[14] Bischetti, M. et al. Widespread QSO-driven outflows in the early Universe. A\&A 630, A59 (2019). 1806.00786

[15] Glikman, E. et al. Luminous WISE-selected Obscured, Unobscured, and Red Quasars in Stripe 82. ApJ 861, 37 (2018). 1805.06961.

[16] Klindt, L., Alexander, D. M., Rosario, D. J., Lusso, E. \& Fotopoulou, S. Fundamental differences in the radio properties of red and blue quasars: evolution strongly favoured over orientation. MNRAS 488, 3109-3128 (2019). 1905. 12108.

[17] Calistro Rivera, G. et al. The multiwavelength properties of red QSOs: Evidence for dusty winds as the origin of QSO reddening. A\&A 649, A102 (2021). 2103.02610.

[18] Glikman, E. et al. FIRST-2MASS Red Quasars: Transitional Objects Emerging from the Dust. ApJ 757, 51 (2012). 1207.2175.

[19] Banerji, M., Alaghband-Zadeh, S., Hewett, P. C. \& McMahon, R. G. Heavily reddened type 1 quasars at z $>2$ - I. Evidence for significant obscured black hole growth at the highest quasar luminosities. MNRAS 447, 3368-3389 (2015). 1501.00815.

[20] Weiß, A. et al. Highly-excited CO emission in APM 08279+5255 at $\mathrm{z}=3.9$. A\&A 467, 955-969 (2007). astro-ph/0702669.

[21] van der Werf, P. P. et al. Black hole accretion and star formation as drivers of gas excitation and chemistry in Markarian 231. A\&A 518, L42 (2010). 1005. 2877.

[22] Stacey, H. R. et al. The rocky road to quiescence: compaction and quenching of quasar host galaxies at $\mathrm{z} \sim 2$. MNRAS 500, 3667-3688 (2021). 2009.01277. 
[23] Hönig, S. F. \& Kishimoto, M. Dusty Winds in Active Galactic Nuclei: Reconciling Observations with Models. ApJL 838, L20 (2017). 1703.07781.

[24] Meijerink, R. \& Spaans, M. Diagnostics of irradiated gas in galaxy nuclei. I. A far-ultraviolet and X-ray dominated region code. $A \& A$ 436, 397-409 (2005). astro-ph/0502454.

[25] Meijerink, R., Spaans, M. \& Israel, F. P. Diagnostics of irradiated dense gas in galaxy nuclei. II. A grid of XDR and PDR models. $A \& A 461,793-811$ (2007). astro-ph/0610360.

[26] Ishibashi, W., Banerji, M. \& Fabian, A. C. AGN radiative feedback in dusty quasar populations. MNRAS 469, 1496-1501 (2017). 1704.03712.

[27] Fiore, F. et al. AGN wind scaling relations and the co-evolution of black holes and galaxies. A\&A 601, A143 (2017). 1702.04507.

[28] Costa, T., Rosdahl, J., Sijacki, D. \& Haehnelt, M. G. Driving gas shells with radiation pressure on dust in radiation-hydrodynamic simulations. MNRAS 473, 4197-4219 (2018). 1703.05766.

[29] Ferrarese, L. \& Merritt, D. A Fundamental Relation between Supermassive Black Holes and Their Host Galaxies. ApJL 539, L9-L12 (2000). astro-ph/0006053.

[30] Genzel, R. et al. Phibss: Molecular Gas, Extinction, Star Formation, and Kinematics in the $\mathrm{z}=$ 1.5 Star-forming Galaxy EGS13011166. ApJ 773, 68 (2013). 1304.0668

[31] Chartas, G. X-Ray Observations of Gravitationally Lensed Quasars: Evidence for a Hidden Quasar Population. ApJ 531, 81-94 (2000). astro-ph/9910299.

[32] Glikman, E. Red Quasars are in a Radiatively Driven Blow-out Phase. Research Notes of the American Astronomical Society 1, 48 (2017).

[33] Ricci, C. et al. The close environments of accreting massive black holes are shaped by radiative feedback. Nature 549, 488-491 (2017). 1709.09651.

[34] McMullin, J. P., Waters, B., Schiebel, D., Young, W. \& Golap, K. CASA Architecture and Applications. In Shaw, R. A., Hill, F. \& Bell, D. J. (eds.) Astronomical Data Analysis Software and Systems XVI, vol. 376 of Astronomical Society of the Pacific Conference Series, 127 (2007). 
[35] Barvainis, R., Alloin, D., Guilloteau, S. \& Antonucci, R. Detection of CO (3-2) Emission at z = 2.64 from the Gravitationally Lensed Quasar MG 0414+0534. ApJL 492, L13-L16 (1998). astro-ph/9710326

[36] Calistro Rivera, G., Lusso, E., Hennawi, J. F. \& Hogg, D. W. AGNfitter: A Bayesian MCMC Approach to Fitting Spectral Energy Distributions of AGNs. ApJ 833, 98 (2016). 1606.05648.

[37] Prevot, M. L., Lequeux, J., Maurice, E., Prevot, L. \& Rocca-Volmerange, B. The typical interstellar extinction in the Small Magellanic Cloud. A\&A 132, 389-392 (1984).

[38] Kochanek, C. S. et al. The CASTLES Survey (1999). URL https://www.cfa.harvard.edu/castles/.

[39] Stacey, H. R. et al. Gravitational lensing reveals extreme dust-obscured star formation in quasar host galaxies. MNRAS 476, 5075-5114 (2018). 1705. 10530 .

[40] Dale, D. A., Helou, G., Contursi, A., Silbermann, N. A. \& Kolhatkar, S. The Infrared Spectral Energy Distribution of Normal Star-forming Galaxies. ApJ 549, 215-227 (2001).

[41] Kennicutt, R. C., Jr. The Global Schmidt Law in Star-forming Galaxies. ApJ 498, 541-552 (1998). astro-ph/9712213.

[42] Riechers, D. A., Walter, F., Carilli, C. L. \& Lewis, G. F. Imaging The Molecular Gas in a z = 3.9 Quasar Host Galaxy at 0".3 Resolution: A Central, Sub-Kiloparsec Scale Star Formation Reservoir in APM 08279+5255. ApJ 690, 463-485 (2009). 0809.0754.

[43] Narayanan, D. \& Krumholz, M. R. A theory for the excitation of CO in star-forming galaxies. MNRAS 442, 1411-1428 (2014). 1401.2998.

[44] Hung, C.-L. et al. What drives the evolution of gas kinematics in star-forming galaxies? MNRAS 482, 5125-5137 (2019). 1806.04233.

[45] Neeleman, M. et al. The Kinematics of $\mathrm{z} \gtrsim 6$ Quasar Host Galaxies. ApJ 911, 141 (2021). 2102.05679 
[46] Madden, S. C. et al. Tracing the total molecular gas in galaxies: [CII] and the CO-dark gas. A\&A 643, A141 (2020). 2009.00649.

[47] Rizzo, F., Vegetti, S., Fraternali, F., Stacey, H. R. \& Powell, D. Dynamical properties of z $~ 4.5$ dusty star-forming galaxies and their connection with local early-type galaxies. MNRAS 507, 3952-3984 (2021). 2102.05671.

[48] Fraternali, F. et al. Fast rotating and low-turbulence discs at $\mathrm{z}=4.5$ : Dynamical evidence of their evolution into local early-type galaxies. A\&A 647, A194 (2021). 2011.05340

[49] Kim, D., Im, M., Glikman, E., Woo, J.-H. \& Urrutia, T. Accretion Rates of Red Quasars from the Hydrogen $\mathrm{P} \beta$ Line. ApJ 812, 66 (2015). 1510.03887.

[50] Fabian, A. C. The obscured growth of massive black holes. MNRAS 308, L39-L43 (1999). astro-ph/9908064

[51] Bieri, R. et al. Outflows driven by quasars in high-redshift galaxies with radiation hydrodynamics. MNRAS 464, 1854-1873 (2017). 1606.06281.

[52] Costa, T., Pakmor, R. \& Springel, V. Powering galactic superwinds with small-scale AGN winds. MNRAS 497, 5229-5255 (2020). 2006. 05997.

[53] Fabian, A. C., Vasudevan, R. V., Mushotzky, R. F., Winter, L. M. \& Reynolds, C. S. Radiation pressure and absorption in AGN: results from a complete unbiased sample from Swift. MNRAS 394, L89-L92 (2009). 0901.0250.

[54] Stacey, H. R., Lafontaine, A. \& McKean, J. P. Smoke on the water: $\mathrm{CO}$ and $\mathrm{H}_{2} \mathrm{O}$ in a circumnuclear disc around a quasar at redshift 2.64. MNRAS 493, 5290-5300 (2020). 2002.07186

[55] King, A. R. Black hole outflows. MNRAS 402, 1516-1522 (2010). 0911. 1639.

[56] Faucher-Giguère, C.-A. \& Quataert, E. The physics of galactic winds driven by active galactic nuclei. MNRAS 425, 605-622 (2012). 1204.2547. 
[57] Chartas, G. et al. Multiphase Powerful Outflows Detected in High-z Quasars. ApJ 920, 24 (2021). 2106.14907.

[58] Dadina, M. et al. Yet another UFO in the X-ray spectrum of a high-z lensed QSO. A\&A 610, L13 (2018). 1801.09839

[59] Sharon, C. E. et al. A Total Molecular Gas Mass Census in z 2-3 Star-forming Galaxies: Low-J CO Excitation Probes of Galaxies’ Evolutionary States. ApJ 827, 18 (2016). 1606.02309

[60] Riechers, D. A. Molecular Gas in Lensed z >2 Quasar Host Galaxies and the Star Formation Law for Galaxies with Luminous Active Galactic Nuclei. ApJ 730, 108 (2011). 1101.5624

[61] Riechers, D. A. et al. $\mathrm{CO}(\mathrm{J}=1 \rightarrow 0)$ in $\mathrm{z}>2$ Quasar Host Galaxies: No Evidence for Extended Molecular Gas Reservoirs. ApJL 739, L32 (2011). 1106.2553

[62] Virtanen, P. et al. SciPy 1.0: Fundamental Algorithms for Scientific Computing in Python. Nature Methods 17, 261-272 (2020).

[63] Harris, C. R. et al. Array programming with NumPy. Nature 585, 357-362 (2020).

[64] Hunter, J. D. Matplotlib: A 2d graphics environment. Computing in Science \& Engineering 9, 90-95 (2007)

[65] Wenger, M. et al. The SIMBAD astronomical database. The CDS reference database for astronomical objects. $A \& A S$ 143, 9-22 (2000). astro-ph/0002110.

[66] Ochsenbein, F. et al. The VizieR database of astronomical catalogues.

[67] Hezaveh, Y. D. et al. ALMA Observations of SPT-discovered, Strongly Lensed, Dusty, Star-forming Galaxies. ApJ 767, 132 (2013). 1303.2722

[68] Spilker, J. S. et al. ALMA Imaging and Gravitational Lens Models of South Pole TelescopeSelected Dusty, Star-Forming Galaxies at High Redshifts. ApJ 826, 112 (2016). 1604.05723. 
[69] Shajib, A. J. et al. STRIDES: a 3.9 per cent measurement of the Hubble constant from the strong lens system DES J0408-5354. MNRAS 494, 6072-6102 (2020). 1910.06306.

[70] Blackburne, J. A., Pooley, D., Rappaport, S. \& Schechter, P. L. Sizes and Temperature Profiles of Quasar Accretion Disks from Chromatic Microlensing. ApJ 729, 34 (2011). 1007. 1665.

[71] Stacey, H. R. \& McKean, J. P. A flux-ratio anomaly in the CO spectral line emission from gravitationally lensed quasar MG J0414+0534. MNRAS 481, L40-L44 (2018). 1808.05571.

[72] Tuan-Anh, P. et al. On the dust and gas components of the $\mathrm{z}=2.8$ gravitationally lensed quasar host RX J0911.4+0551. MNRAS 467, 3513-3524 (2017). 1609.03271.

[73] Sluse, D., Hutsemékers, D., Courbin, F., Meylan, G. \& Wambsganss, J. Microlensing of the broad line region in 17 lensed quasars. $A \& A$ 544, A62 (2012). 1206.0731.

[74] Glikman, E. et al. A highly magnified gravitationally lensed red quasar at $\mathrm{z}=2.5$ with significant flux anomaly: Uncovering a missing population. arXiv e-prints arXiv:1807.05434 (2018). 1807.05434.

[75] Cornachione, M. A. et al. A Microlensing Accretion Disk Size Measurement in the Lensed Quasar WFI 2026-4536. ApJ 895, 125 (2020). 1911.06218.

[76] Bate, N. F. et al. HST imaging of four gravitationally lensed quasars. MNRAS 479, 4796-4814 (2018). 1807.03553

[77] Morgan, N. D. et al. WFI J2026-4536 and WFI J2033-4723: Two New Quadruple Gravitational Lenses. AJ 127, 2617-2630 (2004). astro-ph/0312478.

[78] Wu, X.-B. et al. An ultraluminous quasar with a twelve-billion-solar-mass black hole at redshift 6.30. Nature 518, 512-515 (2015). 1502.07418

[79] Wang, F. et al. Spatially Resolved Interstellar Medium and Highly Excited Dense Molecular Gas in the Most Luminous Quasar at $\mathrm{z}=6.327$. ApJ 880, 2 (2019). 1906.06801.

[80] Carniani, S. et al. Constraints on high-J CO emission lines in $\mathrm{z} \sim 6$ quasars. MNRAS 489, 3939-3952 (2019). 1902.01413. 
409 [81] Leipski, C. et al. Complete Infrared Spectral Energy Distributions of Millimeter Detected Quasars at z > 5. ApJ 772, 103 (2013). 1305.3999.

411 [82] Gallerani, S. et al. X-ray spectroscopy of the $\mathrm{z}=6.4$ quasar SDSS J1148+5251. MNRAS 467, 3590-3597 (2017). 1702.07349.

[87] Feruglio, C. et al. The dense molecular gas in the $\mathrm{z} \sim 6$ QSO SDSS J231038.88+185519.7 resolved by ALMA. A\&A 619, A39 (2018). 1804.05566. 